\title{
A Review on Malaria Eradication: What hope for Nigeria?
}

\author{
${ }^{* 1}$ AMADI, EC \\ Department of Applied and Environmental Biology (Parasilogy Unit), Rivers State University of Science and Technology, Nkpolu, Port \\ Harcourt \\ E-mail_amadihiec@yahoo.com.08033414111
}

\begin{abstract}
Malaria and its transmitting vectors are household names. Malaria which helped Africa from the venomous fangs of colonialism has turned to be the bane of development in tropical countries including Nigeria. The factors which promote prevalence of the disease and its transmission dynamics are well discussed in this review paper. As a corollary to the above, sundry recommendations are made. It is hopeful that Nigerian government and other African countries where malaria and its vectors are endemic would exploit these noble ideas as recommended. Also, if the limited grants by donor agencies are properly utilized coupled with the involvement of the right personnels, it is possible that Nigeria will achieve the eradication of malaria. @ JASEM

http://dx.doi.org/10.4314/jasem.v20i3.32
\end{abstract}

Keywords: Malaria, vectors, eradication, parasite, plasmodium, Anopheles.

Since the world wars, malaria has become a household name. During this period, African continent was known as the "Whiteman's grave". Malaria is one of the oldest and most important communicable diseases known to man. This is so especially in the tropics, where it accounts for over $25 \%$ infant mortality, plus substantial morbidity and mortality in adolescents and adults, particularly expectant mothers and malnourished individuals. Apart from Nigeria, the disease occurs in South America, south-central Asia, and Arabian penisular and Western pacific (Amadi et al, 2011). As at the year 2000, over half a billion people globally were projected as populations at risk for malaria, with about 300 million people actually infected. In recent time, the enormity of morbidity, mortality, high economic and social impacts on human population especially in the rural areas appear to be on the increase (WHO, 2001).

In man, five species of plasmodium are known to cause malaria disease condition, which includes plasmodium falciparum, plasmodium malariae, plasmodium vivax, and plasmodium ovale and plasmodium knowlesi. Plasmodium falciparum is the most common and virulent in Nigeria. It infects humans of all sexes and social classes, with attendant economic impact on the working population. Plasmodium species still ranks the number one disease - causing parasites that kills a child somewhere in the globe every 30 seconds, children, pregnant mothers, people in emergency situations and people living with HIV/AIDs are particularly vulnerable to the parasite. Further, diseases caused by plasmodium species accounts for two - third of all deaths of children under the age of five (5) in Africa.
There are up to 800,000 infantile deaths and a substantial number of miscarriages as well as very low - birth weight babies per year due to diseases caused by plasmodium species (Amadi, et al 2013). Indeed, their common mode of transmission is through the bites of infected female Anopheline mosquitoes. The most common and virulent of the mosquitoes is Anopleles gambiae complex. The preponderance of the mosquitoes and availability of a steady pool of infected persons enhance active transmission and sustenance of malaria in many African countries including Nigeria (WHO, 1997). Transmission can also occur between man and man by blood transfusion and sharing of needles in drug addicts. In P: falciparum and $P$. Ovale, the time of rupture of their infected Rbc is 48 hours, while incubation periods are six and nine days respectively. In $P$. vivax, the time of rupture of infected $\mathrm{Rbc}$ is 48 hours and pre-potency period is eight (8) days, while $P$. malariae has a rupture period of 72 hours of its infected Rbc and pre-potency period of $27-37$ days. The severity and duration of the parasite attack in endemic areas (Nigeria) depend on many factors including nutritional status of the host, virulence of the infecting strain, development of resistance or immunity in the infected hosts, age, occupation and environmental factors. In regions where these parasites are prevalent, people have the disease but due to immunity they have few or no symptoms. Severity of the disease symptoms can also vary depending on the individual's general health.

Although, it is not the position of this paper to give detailed symptoms of the disease, brief mention has to be made. Plasmodium species cause acute fibril illness. The first symptoms are fever, headache, 
chills, vomiting, muscular pain, nausea, back and abdominal pains, increased pulse and respiratory rates. These symptoms may be mild and difficult to recognize as malaria (disease caused by plasmodium). If not treated within 24 hours, $\mathrm{P}$. falciparum can progress to severe illness often leading to death. Children in endemic areas with the severe disease, frequently develop one or more of the following symptoms which include anaemia or cerebral malaria which also occur in adults. Further in adults, multi-organ involvement may be frequent when infected with plasmodium species. In $P$. vivax and $P$. Malariae, clinical relapses may occur weeks to months after the first infection, even if the patient has left the malarious area. These new episodes arise from dormant" larval forms (absent in p. falciparum and $P$. malariae) and special treatment targeted at these larval stages is mandatory for a complete cure (Amadi, et al 2015).

The disease has engaged the attention of public health worker throughout the world for over $3 / 4$ of the century. Although, pragmatic efforts are made to reduce the scourge of the disease, it remains a major health hazard today. In Nigeria, it is not easy to estimate the degree of morbidity and mortality from the disease. Apparently, conservative estimates show that about one million African children under the age of five (5) years die annually of the disease, while current estimates put the annual mortality from the disease among Nigerians as 100,000 per year (Enwonwu 1983). This figure as stated would have increased despite efforts made by government and donor agencies to reduce the prevalence of the disease. Therefore, this current review paper exposes factors militating against eradication of the disease, in addition, proffers solutions to be adopted if eradication of the disease is to be achieved.

Factors Affecting Malaria Eradication $n$ Nigeria : Several factors are known to militate against achieving malaria eradication in Nigeria. These factors are as follows:

(1)Factors relating to the parasite: These include the species or strain of plasmodium and their degree of infectivity to mosquito vectors and man in the area. It is noted, that the dominant malaria parasite in Nigeria is the $P$. falciparum which is highly pathogenic, although, not more pathogenic than P.vivax and P.ovale. These malaria parasites are expected to cause minute injury to the infected mosquito species involved in the transmission of the disease. The endemicity of the disease and the availability of steady pool of parasites and its vectors make continuous transmission easy and possible. This factor ensures active sustenance of the disease all year round within the human population, thereby frustrating costly efforts at the elimination of the disease.

(2) Another factor is that there are large numbers of Anopheles mosquitoes transmitting the disease. There are over 200 known species of Anopheles and these vary in their vectoral competence under different environmental or ecological conditions. Amadi (1990), reported another anopheline mosquito known as Anopheles nili and implicated this species of mosquito to the high prevalence of malaria in parts of Niger Delta, Nigeria. This vector species is believed to come from far-east Africa, probably Congo.

(3) Availability of conducive breeding habitats in Nigeria. Some mosquito vectors breed in brackish water, others prefer freshwater. There are remarkable wide range of suitable habitats that can be exploited for reproduction by malaria vectors. Gillet (1975), cited the case of A. gambae in Africa which is closely associated with human habitation and activity, while other malaria transmitters breed in cool, clear, shaded and permanent water bodies in rural areas relatively undisturbed or undeveloped by man. Recently, in Nigeria, the phenomenon of climate change provides no marked difference between rainy and dry seasons of the year: This allows continuous breeding of mosquito vectors and transmission dynamics of the parasite to humans. In Nigeria, plantains are usually planted near human habitation, in which the axils of such plantains retain water after rainfall. The axils of such plantains are utilized by mosquito vector for breeding, thereby ensuring continuous transmission of the disease throughout the year. Also in most rural areas, there is the habit of keeping pots and other empty containers that can hold water near human habitation. Therefore, these provide additional breeding foci for the mosquito vectors. The terrain of most parts of Nigeria assists in the reproduction of malaria vectors. The flat - table land of most Nigerian communities encourages pools of water with occasional waterlogging due to the nature of the soil. These stagnant pools of water are common features of these areas after heavy rainfall. This no doubt will enhance mosquito breeding and transmission of the disease to the human population.

4. The occupation of Nigerians also account for high malaria infections. Many Nigerians are farmers and fishermen, thus these activities predispose them to various mosquito bites. After fishing, cannoes are kept near human habitation, water accumulate inside these cannoes during rainfall and 
these serve as suitable breeding site for mosquito vectors, leading to high malaria infection.

5. The habit and lifecycle pattern of mosquito vectors also impede eradication measures. Some Anopheles species are indoor feeders while others are outdoor feeders. Some species are usually found around human habitation where they rest after feeding, while others only enter houses for feeding after which they fly away. The lifecycle pattern is unique and involves both mosquito vectors and human hosts. In man, appreciable development of the parasite occurs, later this is sucked up by the mosquito during its blood meal and final development occurs in the vectors. The matured parasites (Sporozoites) move to the salivary gland of the vectors ready for inoculation into the human hosts.

6. The effects of temperature, humidity and rainfall are the most important environmental factors that affect the prevalence of malaria. Temperature and humidity not only affect the lifecycle and behaviour of the mosquito vectors but also the rate of development of the parasite within the vector. For instance, the time taken to complete the development of the parasite within the vectors is doubled when the temperature falls by $10^{\circ} \mathrm{C}$. Also, the pattern of rainfall, in addition, to the topography of the area are paramount in determining the types of breeding sites and possibly the type of mosquito species that will occur. The climatic conditions determine man's clothing, housing and lifestyle patterns which all have a bearing on the degree of exposure to and transmission dynamics of the disease.

7. The concept of antigenic variation is another factor that hinders malaria eradiation in Nigeria. The mechanism of the phenomenon is not yet fully understood. It is noted that most protozoan pathogens like plasmodium change their antigens which occur in 'orders'. Research studies, have shown that these 'orders' occur from one to twenty $(1-20)$. During this antigenic pattern, drugs previously taken would be kept in the memory of the parasite and the previous order during which the drug was taken is changed to another order which implies, that particular drug would be ineffective if taken again when attacked by malaria. This antigenic pattern in malaria has led to the ineffectiveness of many drugs now on sale in the market. Apart from this antigenic patterns, malaria parasite masks itself or mimmic the host antibody, thus the host antibodies will not recognize the parasite as non - self, hence the parasite evades the action of the host antibodies.
8. Poverty in many infected individuals impede eradication of malaria. These infected individuals may not have adequate finance for systematic or complete treatment. This sustains pool of parasites in the human population.

9. Illiteracy is another major problem of malaria eradication. Enlightenment of the citizens matters alot when it comes to the eradication of malaria. For instance, due to illiteracy an individual might abuse the use of anti-malaria drugs and time of taken the drugs. This implies, that there will be high level of drug abuse because the individual does not know the correct dosage of the anti-malaria drug to be taking especially in rural areas. It is noted that in rural areas, they do not know how to tackle the issue of destroying breeding sites of mosquito vectors and house to house enlightenment for the elimination of mosquito vectors and parasite transmitted by it. Therefore, low illiteracy, inadequate public enlightenment especially at rural areas and poor health education of the population, combine to make malaria eradication in Nigeria less effective. It has been documented that three major ethnic groups Yoruba, Ibo, Hausa, have made more reasonable efforts at eliminating the disease. Poor compliance with treatment dosages constitutes a major factor in treatment failures and this has implications in increasing occurrence and spread of drug resistance strains of malaria parasite among the infected human population (Olubunmi, 2010).

10. Limited supply of insecticide treated bednets (ITNs). In Nigeria, some states are richer than others, as such the level at which the government in such states will take care of their indigenes as per healthy subsidy will not be the same as it is in other states. For instance, in Rivers State, the government spends huge sum of money annually for the eradication of malaria by sharing insecticide treated bednets. However, this when compared to states like Kwara State where their state allocation is minimal, the supply of these bednets becomes difficult for malaria eradication.

11. Low immunity also raises threat to malaria eradication. It is discovered that children between 15 years had the highest record of the disease as reported in several studies. It is believed that children have low immunity to the disease than adults. This is consistent with the findings of Pant et al (2007), who reported age-related pattern of biting and transmission of the disease. This was attributed to the host preference by the vector, level of immunological responses of the host including differential activities of the age groups. 
12. Another factor impeding malaria eradication in Nigeria is that malaria parasite exhibit the concept of premunition, which is immunity in the presence of an infective agent. As a result of this, all healthy individuals should be diagnosed of the disease, followed by complete treatment of the infection.

13. Inadequate and improper diagnosis of the disease is another important factor. Although many doctors routinely prescribe antimalarial drugs to patients not minding that many disease agents present primary symptoms of malaria. This makes prescriptions by doctors ineffective, thus ensuring persistence of the disease in the infected individuals.

14. There is the question of drug choices, their relative efficacies and the matter of cost to be incurred by generality of the people (Gilles, 1987). Available records, show that cost of certain quality drugs have tended to predispose infected human population to patronize quacks for cheaper, sometimes fake or expired drugs. Sincerely, NAFDAC is making efforts at eliminating fake or substandard drugs on sale in the market which will reduce the prevalence of malaria including other diseases in human population

15. The issue of drug resistance by some species of plasmodium parasites as cited by Pyne (1987) has made eradication by drug treatment futile. The increasing incidence of drug resistance cases in malaria patients, certainly poses a major problem to future management or eradication of the disease (Basco et al, 1993). In addition to drug resistance is the emergence of pesticide resistance by Anopheles species which increases its difficulty and possible elimination of the vectors.

16. The pathology or clinical symptoms of malaria - acute or chronic phases, require proper understanding to help improve better management or eradication of the disease and its complications. Indeed, the need for improved skills, expertise and technical facilities cannot be over - emphasized (Janssen's and Wory 1987). The involvement of parasitologists, medical entomologists, medical doctors and other public health workers are vital in achieving proper eradication of the disease. The above is lacking in Nigeria, thereby impeding on the disease eradication. Further, is the problem of inadequate planning and poor execution of vector control or management. The issues of logistics, proper funding and involvement of skilled and choice of pesticides need to be properly understood. Also, lack of adequate knowledge of the detailed ecology especially behavioural characteristics population dynamics and natural history of the Anopheles vectors are major setback to the possible eradication of the disease. This accounts for the failure of various control efforts of which are founded on faulty criteria (WHO, 1986). However, malaria eradication in Nigeria faces cloudy policy objectives and most times, wrongful orientation of control efforts or eradication plans, programmes and effective operations. There is need to involve experts to achieve proper eradication of the disease.

In conclusion, the fight against malaria, receives attention by the day. More innovative tool for control or management skill still utilizes the fundamental method of chemical, biological and physical measures. We will continue to witness innovative and endless merchandize in the market until we conquer this devastating alien. The mosquito that saved our land from colonization has turned its guns (proboscis) against us. The struggle against infective mosquitoes and malaria will continue as most of us battle with mosquitoes every night with all types of tools. Recently, Nigeria has adopted malaria policy as stipulated by WHO, that herbal remedies form an integral part of the disease management in our society. The nature, quality, usage and diversity of the herbal remedies in use for malaria treatment in various local government areas and cities are not well documented.

Prospects of Malaria Eradication in Nigeria: The possible eradication of malaria in Nigeria has continued to prove difficult and near frustration. There is cause for hope in dealing with the disease in the years to come. This hope is founded on the following factors;

(1) The past few years has witnessed a high upsurge in the efforts to sanitize our environment. To achieve this, the need for active drainage of gutters and destruction of breeding sites of mosquitoes has become gradually inculcated in the human populations in Nigeria.

(2) Malaria treatment using a single dose choice of drugs of proven efficacy is becoming more widely accepted by the majority of Nigerians. This is in addition to the use of suitable chemotherapeutic drugs for sustained reduction of the infection and more skilled handling of complicated cases (including those of drug resistance) using recommended combinations of quality drugs.

(3) Another area of encouragement relates to vector control through the use of insecticide 
impregnated bed nets and the selective treatment of mosquito resting and breeding sites with choice insecticides and larvicides. The increasing use of biological, genetic and other alternative control efforts for malaria vector elimination, also give reasonable hope for the reduction of vector population (Cutis, 1994).

(4) Of great promise is the progress to be made in the development of vaccine for proper elimination of malaria parasites (Waine and McManus 1995). It is noted that some test trials of vaccines has yielded positive results, therefore, providing the way for possible pilot - scale trials of some malaria vaccine before the end of the century. Recently in Nigeria, the minister for Health during World Malaria Day (2016), announced the adoption or development of vaccine for the elimination of malaria disease. It is hopeful that this agenda would be pursued with vigour if Nigeria is to make reasonable breakthrough in the elimination of malaria.

(5) Malaria elimination will benefit from contemporary global advancement in the strategies and methods available for communicable disease management. Such noble achievement is possible through sundry researches and development initiatives relating to malaria diagnosis, immunology, epidemiology and treatment through chemotherapy etc. Further, malaria and other communicable diseases elimination will gain from the revolutionary advancements in communication technology and concomitant polarization of mass media usage for public enlightenment and mass mobilization. Therefore, these present enormous opportunities for grassroots involvement and possible ultimate eradication of malaria and other communicable diseases.

(6) Indeed, there is bold WHO-sponsored global initiative known as Roll-Back-Malaria programme, which has been adopted by most tropical African countries including Nigeria. The programme targets effective measures and application of operations research for achieving substantial reduction and possible elimination of malaria. The programme targets $50 \%$ reduction of overall malaria deaths by the 2010 and the remaining $50 \%$ by the year 2025 . Some opportunities are still available for Nigeria in achieving eradication of malaria based on the above premise.

Recommendations: Since some parts of Nigeria has proper drainage system, good sanitation is necessary to reduce mosquito breeding sites. Government should provide drainage system which will drain out all the stagnant water after heavy rainfall. The current monthly environmental sanitation should be pursued with much vigour, at present the exercise is weighing down in its potency.

There should be proper diagnosis of the disease, since many other disease agents show primary symptoms of malaria e.g. typhoid. It is possible for diagnosis of the disease to be undertaken at the immunological or biochemistry level to possibly distinguish malaria diseases from symptoms of other pathogens.

There should be prompt administration of appropriate anti-malaria agents, early recognition of complications and integrated control method which will result in appreciable decline of transmission dynamics of the malaria parasite.

The use of insecticide treated bednets should be compulsory and available to rural and urban population. To achieve this, adequate campaigns must be mounted in rural and urban areas of Nigeria. This will create awareness and better understanding on the benefits of ITNS. However, the limited number of ITNs is a major problem. Therefore, many rural and urban dwellers do not have access to ITNs.

There should be proper understanding of the vector and the disease dynamics which are vital for successful elimination of malaria and its vector.

There should be total aerial spraying of insecticide including stagnant waters. The insecticide to be used for this purpose, should have a selective killing capacity of the mosquito vector. This implies that this insecticide should be specially manufactured so that beneficial insects will not be killed in the process. Although, this is difficult to achieve but was adopted by countries that have eradicated malaria like Poland, New-Zealand, America, Britain and Bangladesh. Further, it is advocated that quantization of infected people with malaria should be adopted. This is again a difficult task which cannot be achieved easily, considering our cultural, behavioural and other vital characteristics.

Government should make concerted effort for regular medical checkup of its citizens. Although, at the rural level, this is not achievable. The primary health centres saddled with this function are inadequate coupled with shortage of medical personnel's. In addition to this, many urban dwellers do not recognize the disease as life - threatening as most often deaf ears are paid to any campaign relating to malaria. 
Conclusion The scourge of malaria parasite has remained a major unsolved problem in tropical Africa especially Nigeria, that has high and stable malaria transmission. Priority attention has been given to the disease by WHO, yet the menace of the disease remains unabated. Many control efforts have been mounted by various international agencies involved in the eradication of the disease, but it still persists. It is hopeful that the current effort by the World Bank in the reduction of the disease prevalence will be properly applied in order to achieve the desired result. As a result of some or combined factors already discussed, eradication of the disease has remained a mirage in the country. It is believed, that adopting the aforementioned recommendations, the rate of malaria transmission will drastically be reduced to a bearable minimum. Further, it is also believed that the chances do exist for effective control and possible eradication of the disease in the years to come, given the political will, adequate funding, executive capacity and collective involvement of medical and paramedical personnels.

\section{REFERENCES}

Amadi EC, Harry, SI, and Ogbalu, OK (2015). A Preliminary Survey on the Abundance of Mosquito Species and Transmission of Plasmodium to Humans in Bille, Oporoama, Sama and Tema Communities of Rivers State, Nigeria. Int. Org. Sci. Res./ J. pharm. Bio. Sci. (IOSR-JPBS), 10(6), $82-86$.

Amadi, EC (1990). Survey of Common Human Parasitic Infections in Asari-Toru Local Government Area of Rivers, Nigeria (M.Sc Thes. Unpubl. $1-4$.

Amadi, EC, Sunday, HJ and Usip, LPE (2011). Prevalence of Malaria Parasite in Hospitals of Port Harcourt Metropolis, Rivers State Nigeria. Nig. J. parasitol. 32(2): $215-219$.

Curtis, CF (1994). Case for Malaria Control by Genetic Manipulation of its Vector, Parasitol. Today 10:371 - 372 .
Enwonwu, CO (1983). New Enzyme in Malaria Parasite. Nat. Conc. 3:(393):4.

Gillet, JD (1975). Mosquito-Borne Disease - A Strategy of the Future. John Swain and Company Ltd, London 1:103.

Iwuala, MOE (2001). Malaria Control: The Problems and Prospects. Int. J. Envital. Hlth. Hum. Dev. 2(2): $1-4$.

Janscens, PG Wory, M (1987). Malaria in Africa, South of the Sahara. Ann. Trop. Med. Parasitol. $8(15): 487-498$.

Olubunmi, AA (2010). Perpetual War Against Parasites. A Research Excursion. Uni. Lag. Inaug. Lect. $39-40$.

Pant, CP, Rishikesh, N, Bang, YH and Smith, A (1981). Progress in Malaria Vector Control. Bull. WHO 59(3):305 - 492.

Pyne, D (1987). Spread of Chloroquine Resistance in Plasmodium falciparum, Parasitol. Today: 3241 $-246$.

Waine, GJ and McManus, DP (1995). Nucleic Acids: Vaccines for the Future. Parasitol. Today, 11:113 - 116 .

WHO,(1986). Resistance of Vectors and Reserviours of Diseases to Pesticides. WHO/Tech. Rep. Ser. 88 .

WHO,(1997). Environment and Disease WHO/TDR News no 53 (June, 1997), $1-3$.

WHO,(2001). WHO Recommended Strategies for the Prevention and Control of Communicable Diseases. WHO /TCDLS /CPE /SMT /2017/13. 107-110 